\title{
Characterization of a gene controlling heterocyst differentiation in the cyanobacterium Anabaena 7120
}

\author{
William J. Buikema and Robert Haselkorn \\ Department of Molecular Genetics and Cell Biology, University of Chicago, Chicago, Illinois 60637 USA
}

\begin{abstract}
Anabaena 7120 mutant 216 fails to differentiate heterocysts. We previously identified a 2.4-kb wild-type DNA fragment able to complement this mutant. We show here that the sequence of this fragment contains a single open reading frame (het $R$ ), encoding a 299-amino-acid protein. Conjugation of deletion subclones of this fragment into strain 216 showed that the het $R$-coding region is both necessary and sufficient for complementation of the Het ${ }^{-}$phenotype. The mutation in 216 is located at nucleotide 535 in the hetR gene, converting a serine at position 179 in the wild-type protein to an asparagine in the mutant. Interruption of the het $R$ gene in wild-type cells results in a mutant phenotype identical to that of 216 . Both 216 and wild-type cells containing wild-type het $R$ on a plasmid display increased frequency of heterocysts, even on media containing fixed nitrogen. These results suggest that het $R$ encodes a product that is not only essential for but also controls heterocyst development. This putative regulatory protein lacks known structural motifs characteristic of transcription factors and probably acts at a level one or more steps removed from its target genes.
\end{abstract}

[Key Words: Cyanobacteria; heterocyst; nitrogen fixation; Anabaena 7120; regulation; development]

Received December 7, 1990; accepted December 28, 1990.

Cyanobacteria are a diverse family of prokaryotes that carry out oxygenic photosynthesis similar to green plants. Filamentous cyanobacteria grow in long chains of identical vegetative cells when combined nitrogen is available. When fixed nitrogen is limiting, many strains of filamentous cyanobacteria respond by activating genes encoding the subunits of nitrogenase. In heterocystous cyanobacteria such as Anabaena 7120 , the filaments also undergo patterned cellular differentiation in response to nitrogen limitation. Terminally differentiated cells called heterocysts develop at intervals along the filament, providing the sites at which nitrogen fixation occurs. Heterocysts differ from vegetative cells in a number of functionally important ways. To limit oxygen entry and protect nitrogenase from inactivation, developing heterocysts (proheterocysts) form a new external cell envelope containing inner glycolipid and outer polysaccharide layers. Other structural changes include the reorganization of photosynthetic membranes and the formation of specialized junctions with neighboring vegetative cells. Many biochemical changes occur in the heterocyst, including the inactivation of ribulose bisphosphate carboxylase and the oxygen-evolving photosystem II (PSII) photochemical reaction center complex, and the induction of genes involved in nitrogen fixation and its support, such as the oxidative pentose pathway and a heterocyst-specific ferredoxin /Winkenbach and
Wolk 1973; Haselkorn 1978; Stewart 1980; Wolk 1982; Carr 1983; Böhme and Haselkorn 1988).

Changes in intercellular communication are also apparent as a consequence of heterocyst differentiation. In differentiated filaments, carbon compounds from neighboring vegetative cells are transported into heterocysts (Wolk 1968). Glutamine, carrying newly fixed nitrogen in its amide group, is transported from heterocysts to vegetative cells (Meeks et al. 1978). In Anabaena 7120, heterocysts are spaced approximately 10-20 cells apart, depending on growth conditions. Heterocysts inhibit the differentiation of adjacent vegetative cells, and as the vegetative interval lengthens new heterocysts form midway between existing ones (Wilcox et al. 1973a). Thus, the heterocyst spacing pattern is maintained during growth on $\mathrm{N}_{2}$ as nitrogen source. Transport and metabolism of a specific nitrogenous compound such as glutamine has been proposed as the source of this pattern (Haselkorn 1978), but this is unlikely in light of the normal heterocyst spacing patterns that are seen in most mutants defective in nitrogen fixation (W.J. Buikema and R. Haselkorn, unpubl.; Wilcox et al. 1975).

The formation and maintenance of heterocysts is strongly influenced by neighboring vegetative cells and growth conditions. A classic study by Wilcox et al. using filament breakage showed that proheterocysts revert at higher frequencies and at later stages when fewer adja- 
cent vegetative cells are present to support them (Wilcox et al. 1973b). This is not surprising considering that vegetative cells act not only as a sink for fixed nitrogen from the heterocysts, but also as the source of fixed carbon to the heterocysts. This carbon serves not only as an energy and reductant source for nitrogen fixation, but as a substrate for the addition of ammonia from the nitrogenase enzyme complex.

Earlier we described the isolation and characterization of chemically induced $\mathrm{Fix}^{-}$mutants of Anabaena 7120 , including the complementation of a strain defective in an early step of heterocyst differentiation (Buikema and Haselkorn 1991). Here we describe the characterization of the hetR gene of Anabaena 7120 that is required for this early step and is capable of inducing multiple adjacent heterocysts when present on a plasmid in wild-type cells.

\section{Results}

Sequence of the hetR gene

Earlier we had identified and cloned a $2.4-\mathrm{kb}$ partial Sau3AI fragment of wild-type DNA able to complement the heterocyst-deficient mutant 216 (Buikema and Haselkorn 1991). A physical map of this fragment is shown in Figure 1. The DNA sequence of the entire 2.4-kb fragment was determined and is shown in Figure 2. The sequence contains one complete open reading frame (ORF) capable of encoding a 299 -amino-acid protein of $35 \mathrm{kD}$, and no transcription termination structures are evident immediatcly downstream of the ORF. No significant similarities were found using the FASTA program (Pearson and Lipman 1988) to compare the DNA sequence of het $R$ to GenBank (release 64 ), or its predicted protein

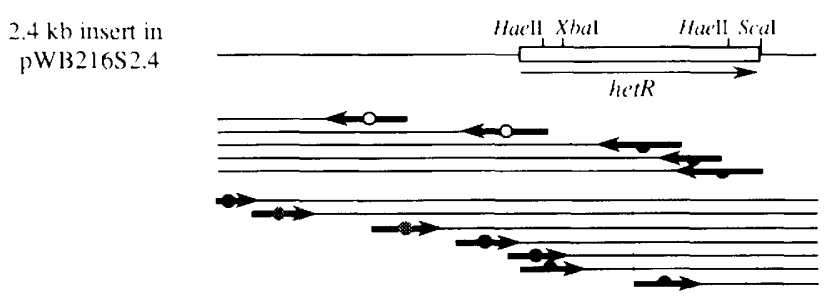

Figure 1. Physical map of the $2.4-\mathrm{kb}$ insert in plasmid pWB216S2.4 containing the het $R$ region. Only selected restriction sites are indicated. Wide horizontal lines indicate the portions of the nested deletions that were sequenced. The remainder of each line indicates the DNA remaining in that deletion. These deletions were used for complementation tests. If a deletion complemented, the line is marked with a filled circle. The two deletions that complemented poorly are shown marked with stippled circles. Deletions that produced only a few marker-rescued colonies are shown marked with a half-circle. Lines marked with open circles indicate deletions that neither complemented nor gave marker-rescued colonies. The markerrescue results place the mutation in strain 216 within the 160 bp region shown between the vertical dotted lines. Other deletions used for sequencing but not for complementation tests are not included in the figure. sequence to GenPept (release 64), the translated protein reading frames from GenBank. The expected gene product shows no evidence of extended hydrophobic regions indicative of a signal sequence or membrane localization. The protein contains over $30 \%$ charged residues $(92$ of 299), although the net charge is only -6 , giving an expected $\mathrm{pI}$ of 6.37 . The codon usage of het $R$ when compared to highly expressed Anabaena 7120 genes suggests that it is expressed at a low to moderate level (data not shown). Secondary structure prediction algorithms (Chou and Fasman 1978; Garnier et al. 1978) suggest a predominance of $\alpha$-helical over $\beta$-sheet structures. The het $R$ peptide sequence does not contain significant similarities to common DNA-binding motifs like helixturn-helix, zinc finger, or helix-loop-helix, as documented in the PROSITE database (Bairoch 1989).

\section{Complementation of mutant 216 using deletion subclones}

We sought to determine the minimum genetic requirement for complementation of strain 216 by using some of the deletions constructed for DNA sequencing. Selected deletions were subcloned into the polylinker of the shuttle vector pCCB110 /described in Materials and methods), and the resultant plasmids were transferred via conjugation into strain 216 . Figure 1 shows the deletions that were used in this analysis and indicates those that complement 216 . The entire ORF is necessary and sufficient for efficient complementation of the mutation in strain 216. Some reduction of complementation efficiency was seen with the two farthest upstream deletions that lacked 270 and 730 bases of pWB216S2.4, but the reason for this is unknown. These deletions may have fused a vector promoter to the het $R$ gene, resulting in a lethal phenotype (see below).

After determining which deletion subclones could still complement strain 216, we observed that the introduction of other subclones with larger deletions resulted in small numbers of $\mathrm{Fix}^{+}$colonies on $\mathrm{N}^{-}$plates after several weeks. It seemed likely that these colonies were the result of recombination between the introduced plasmid and the mutant chromosomal het $R$ gene. We used the frequency of this marker rescue by recombination (as reflected in the number of $\mathrm{Fix}^{+}$colonies) together with the end points of those deletion subclones that did not form these colonies to locate the chromosomal lesion in strain 216 (data not shown). These results mapped the mutation to a 160-bp region near the center of the het $R$ gene, as indicated in Figure 1.

A 318-bp region containing the expected mutation in 216 DNA and the corresponding region of wild-type DNA were amplified using the polymerase chain reaction (PCR). The sequences corresponding to the oligonucleotide primers used are shown in Figure 2. Following symmetric amplification, a portion of the doublestranded product was amplified asymmetrically, and the single-stranded product was sequenced. A single nucleotide difference was detected between wild-type and 216 DNA in this region. The guanine at position 1687 in 
241

2161 GCAGCGATGGGCGGTTTTAACTGCTAAAAATTCAGGATTTGATCGCTCCTTTGGCGGCGATCGTTTATATCAATCAAACTGGTCAAGTCCCTAGTGAT 2258

Figure 2. Annotated nucleotide sequence of het $R$ and flanking regions and deduced amino acid sequence of the het $R$ gene product The first available methionine codon in the het $R$ ORF was chosen as the start of translation. The underlined nucleotides indicate the sequences used for priming the PCR employed to identify the mutation in strain 216 . The mutated residue at position 1687 is indicated in boldface, as is the wild-type serine that is replaced by asparagine in strain 216.

Figure 2 is changed to an adenine in strain 216 , resulting in a single amino acid change in the predicted het $R$ gene product of serine (wild-type) to asparagine (mutant 216).

\section{Insertion cassette mutagenesis of hetR}

To confirm that inactivation of the hetR ORF is sufficient to cause the observed phenotype in strain 216 , we constructed a mutation of het $R$ in wild-type Anabaena 7120 by insertion of a neomycin-resistance cassette derived from Tn 5 as described in Materials and methods. Several independent colonies were isolated and characterized. Every colony showed rapid bleaching and a complete lack of heterocysts when grown under nitrogenlimiting conditions, identical to the phenotype of strain 216. One of these recombinants, WJB313, was confirmed to have the expected genomic arrangement of a single interrupted het $R$ gene by Southern hybridization, using the 700-bp HaeII fragment internal to het $R$ as probe (data not shown). This het $R$ mutant strain grows as well as wild-type and mutant 216 on media containing nitrate or ammonia. Thus, the het $R$ gene does not appear to play a significant role in normal vegetative growth.

\section{Supernumerary heterocysts}

In the course of examining strain 216 carrying the complementing wild-type copy of the het $R$ gene in pWB216S2.4, we observed an unusually high frequency of heterocysts in this strain when it was grown under $\mathrm{N}^{-}$conditions. Many of these heterocysts were present in multiples, mostly as pairs but some with as many as five contiguous heterocysts. Typically $10-25 \%$ of the total number of cells under these conditions were heterocysts. To confirm that this result is due to the het $R$ gene itself, we examined the phenotypes of 216 carrying each of the deletion derivatives of pWB216S2.4 that had been used to test for complementation of the $\mathrm{Het}^{-}$pheno- 
type. In all but one of the cases in which the ORF remained intact, the extra heterocyst phenotype was seen under $\mathrm{N}^{-}$conditions. The lone exception was a deletion that removed all but 50 bp of DNA upstream to the het $R$ ORF. This plasmid was able to complement 216 , but showed a normal number of heterocysts under $\mathrm{N}^{-}$conditions. Presumably this deletion derivative expresses the plasmid copy of het $R$ at a low enough level to avoid generating excess heterocysts, but at a level high enough to allow complementation.

We also examined the phenotypes of wild-type Anabaena 7120 carrying each of the deletion plasmids previously tested in 216 . The results paralleled those from 216 with the difference that, since the wild-type strain is $\mathrm{Het}^{+}$, normal numbers of heterocysts were seen with plasmids deleted into the het $R$ ORF from either end. The latter result indicates that the extra heterocyst phenotype is not due to the titration of a factor that binds to DNA outside the hetR ORF. Figure 3 shows the typical supernumerary heterocyst phenotype displayed by wildtype Anabaena 7120 carrying pWB216S2.4. In this strain grown with nitrate, approximately $5 \%$ of the cells look like heterocysts (Fig. 3A). These cells are not as distinct as normal heterocysts and are not functional based on acetylene reduction assays (data not shown). Under $\mathrm{N}^{-}$ conditions, many groups of multiple heterocysts are seen, most of which occur as pairs, but occasionally as groups of three, four, or more cells (Fig. 3B,C). The heterocyst pairs are usually similar in size and appearance, suggesting that they develop synchronously. However,
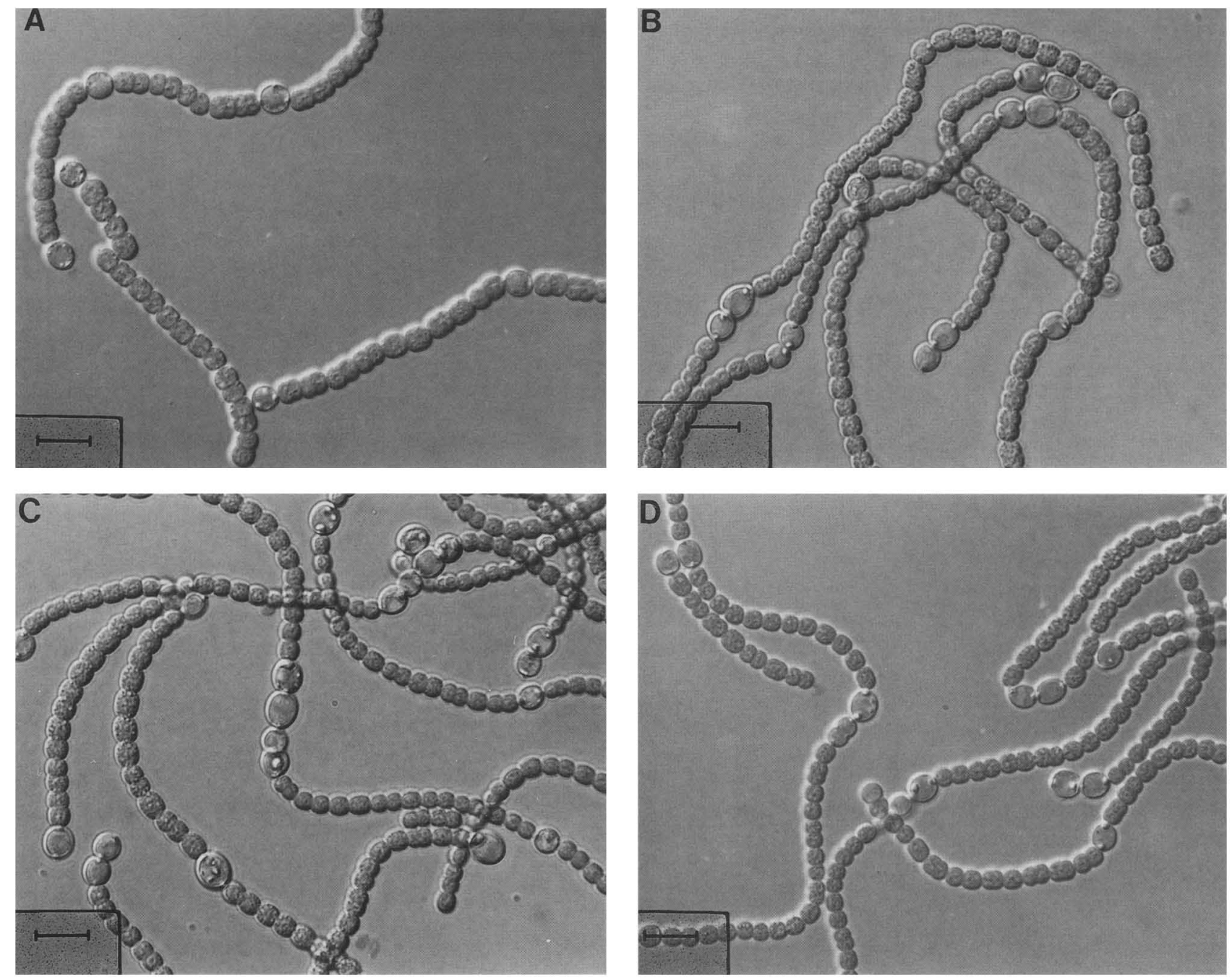

Figure 3. Light microscopy of Anabaena 7120 containing a plasmid carrying the hetR gene. Anabaena 7120 carrying pWB216S2.4 was grown on BG-11 plates with nitrate $|A|$ or without combined nitrogen $(B, C, D)$. Note the presence of heterocysts in $A$. Wild-type filaments grown on nitrate typically develop less than $1 \%$ heterocysts. In $B, C$, and $D$ many groups of heterocysts are seen, and the heterocyst frequency is increased with respect to wild-type. $B$ shows the typical appearance of Anabaena containing het $R$ on a plasmid. Most of the heterocysts present are single or paired cells. Occasionally higher multiples are seen, as shown in $C$. Often the heterocysts in these groups have different sizes, indicating that the group formed asynchronously, and one or more of the heterocysts typically appears abnormal. Rarely, heterocysts are seen that appear to be dividing, as shown in $D$ (left center). Bar, $10 \mu \mathrm{m}$. 
the higher multiple groups vary in size, often with one or more of the cells appearing abnormal, suggesting that the group develops asynchronously. Occasionally pairs of heterocysts are seen in which the two cells are incompletely separated or appear to be dividing (Fig. 3D). The appearance of these heterocysts suggests that dividing cells may occasionally be induced to form heterocysts before cell division is complete.

The excess heterocyst phenotype of Anabaena strains containing a plasmid with an intact copy of the het $R$ gene, such as pWB216S2.4, was typically unstable. Older cultures showed a high frequency of plasmid loss. When maintained on $\mathrm{N}^{+}$plates, strain 216 carrying pWB216S2.4 often formed colonies that were Fix ${ }^{-}$Het $^{-}$ on return to $\mathrm{N}^{-}$medium. A continuous liquid culture of wild-type Anabaena 7120 containing pWB216S2.4 grown for 3 weeks under neomycin selection with ammonia as nitrogen source yielded approximately $50 \%$ Fix $^{-}$Het $^{-}$mutant colonies. Anabaena 7120 containing pWB216S2.4 grows more slowly on selective plates with fixed nitrogen than strains containing an inactive het $R$ plasmid, and grows very slowly on selective $\mathrm{N}^{-}$plates. Although the nature of these mutations is being studied further, it is likely that the presence of extra copies of het $R$ results in a strong depression of growth rate. Inappropriately formed heterocysts directly reduce the growth rate of filaments by being terminally differentiated, and indirectly by acting as a sink for fixed carbon from neighboring cells. Thus, it is probable that mutations, gene conversions, or recombination events that inactivate the extra copies of het $R$ or other genes required for differentiation are selected under these conditions.

\section{Expression of hetR}

The timing and level of expression of the het $R$ gene was determined by Northern blot analysis. Total RNA was prepared from wild-type Anabaena 7120 at 6-hr intervals after induction by removal of fixed nitrogen. A blot of this RNA was probed with the internal $0.7-\mathrm{kb}$ HaeII fragment of the het $R$ gene (see Fig. 1) with the results shown in Figure 4. At zero time, only weak hybridization is seen in two bands of $1.4 \mathrm{~kb}$ and $1.9 \mathrm{~kb}$, indicating that het $R$ is transcribed at a low level during growth under nitrogenreplete conditions. From 6 to $18 \mathrm{hr}$ post-induction, the level of het $R$ RNA increases from three- to fivefold, decreasing slightly after $18 \mathrm{hr}$. Both the $1.4-\mathrm{kb}$ and $1.9-\mathrm{kb}$ bands increase uniformly in intensity, and a very faint band of $2.4 \mathrm{~kb}$ appears. This result indicates that induction of het $R$ occurs very early during heterocyst development.

The presence of multiple bands on the Northern blot suggests that het $R$ is transcribed from multiple promoters or that the mRNA is processed. The $1.4-\mathrm{kb}$ and $1.9-\mathrm{kb}$ major transcripts contain $0.5 \mathrm{~kb}$ and $1.0 \mathrm{~kb}$ more than is necessary to encode the 900-bp hetR ORF. Because no significant ORFs are apparent within $1 \mathrm{~kb}$ upstream of $h e t R$, it is possible that both transcripts originate from this region without encoding additional genes. Although

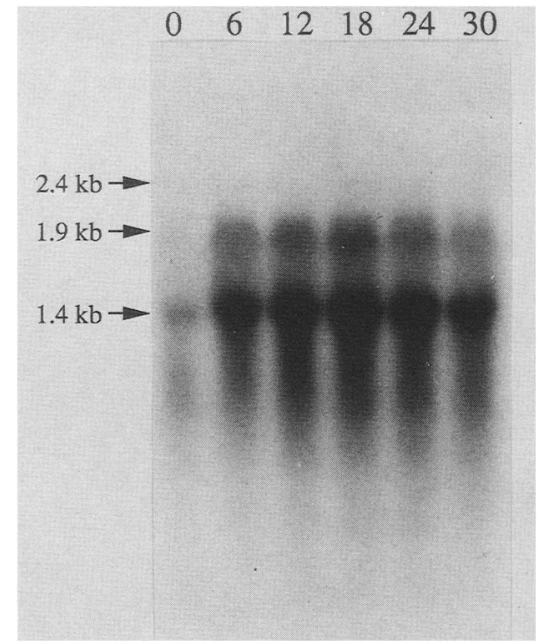

Figure 4. Hybridization of het $R$ to total RNA isolated from Anabaena 7120 induced by nitrogen starvation. The 700-bp Haell fragment internal to hetR (Fig. 1) was used to probe a Northern blot of RNA isolated from Anabaena 7120 induced for heterocyst differentiation at the times shown (in hours). Sizes are based on glyoxalated RNA standards.

this possibility requires very long $5^{\prime}$-untranslated regions, such lengths have been observed in other Anabaena transcripts, such as the rbcLS operon (NierzwickiBauer et al. 1984) and the Anabaena 7120 sigA gene, for which B. Brahamsha has mapped the $5^{\prime}$ end of one transcript approximately 800 bp upstream of the gene (B. Brahamsha, unpubl.). Alternatively, the transcripts may extend downstream from het $R$. No additional ORFs are present in the 200 bp $3^{\prime}$ to het $R$ that have been sequenced.

\section{Distribution of het $\mathrm{R}$ in cyanobacteria}

As a means of correlating the presence of het $R$ with heterocyst formation, it was of interest to determine the distribution of the het $R$ gene in various groups of cyanobacteria. An internal 0.7-kb HaeII fragment (Fig. 1) of the het $R$ gene was used to probe a Southern blot of chromosomal DNAs from 24 strains of cyanobacteria, under conditions of high stringency. We included heterocystous, filamentous nonheterocystous nitrogen-fixing, and single-celled nonfixing strains in the comparison. As shown in Figure 5, DNA hybridizing to the het $R$ gene is present in all filamentous cyanobacteria examined but is not present in the two single-celled cyanobacterial strains examined. To test for nitrogen fixation ability in the tested strains, the blot was reprobed with the nif $H$ gene region of Anabaena 7120 found in pAn 154.3 (Rice et al. 1982). The presence of a het $R$ analog in all of the filamentous strains coincides with the presence of DNA hybridizing to the nif $H$ gene. Conversely, no hybridization to nifH was seen for the single-celled strains /data not shown).

In general, the intensity of hybridization to the het $R$ 


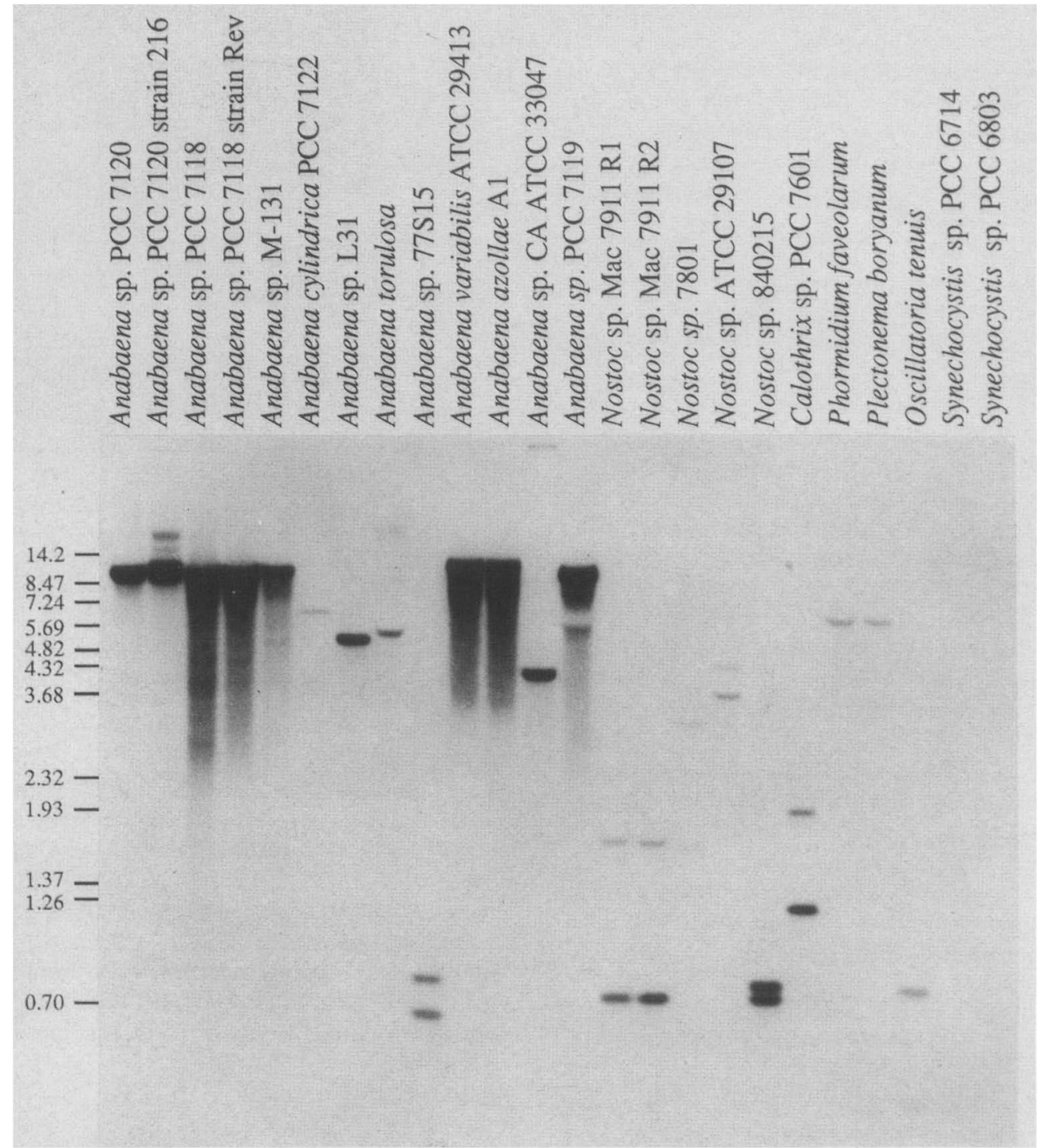

Figure 5. Hybridization of hetR to genomic DNA from several classes of cyanobacteria. Approximately $2 \mu \mathrm{g}$ of genomic DNA restricted with $H$ indIII was used from each of the indicated cyanobacterial strains, and the Southern blot was probed with the 700 -bp HaeII fragment internal to het $R$ (Fig. 1). The smears seen in several lanes are caused by partially sheared DNA samples. With the exception of Anabaena 7118, all of the Anabaena, Nostoc, and Calothrix strains are filamentous heterocystous cyanobacteria. The Phormidium, Plectonema, and Oscillatoria strains are filamentous, nonheterocystous, anaerobically nitrogen-fixing cyanobacteria. The two Synechocystis strains are unicellular non-nitrogen-fixing cyanobacteria.

probe is correlated with the relatedness of the strains examined to Anabaena 7120 . Strains that are nearly identical to 7120 , such as 7118,7119 , and M-131, show the strongest hybridization and similarly sized bands. More distantly related cyanobacteria, such as the nonheterocystous Phormidium, Plectonema, and Oscillatoria strains, show a lower level of hybridization and variable band sizes. In these cyanobacteria, a het $R$ analog cannot be involved in heterocyst formation, but it may function in the patterned development of nitrogen-fixing cells. Further work is required to determine if het $R$ analogs always accompany nitrogen fixation capability, both by expanding the number of strains tested and by testing other categories of cyanobacteria, such as filamentous non-nitrogen-fixing species and single-celled nitrogen-fixing species.

\section{Discussion}

The het $R$ gene appears to be a novel regulatory gene. It contains no sequence similarity to rpoD or any other sigma factor-like genes from Anabaena (B. Brahamsha, pers. comm.). The het $R$ protein sequence contains no apparent DNA-binding domains, such as zinc finger or helix-turn-helix, indicative of transcriptional activators. There is no sequence similarity to the protein ki- 
nases or their DNA-binding substrates that activate many environment-sensitive genes in bacteria (Kustu et al. 1989). There have been no difficulties in subcloning the het $R$ gene in Escherichia coli, suggesting that there is no interaction between its product and the transcription apparatus of $E$. coli. The identification of the probable mutation of the het $R$ product in strain 216 resulting in a change from serine to asparagine may be useful in the eventual determination of its function.

The het $R$ gene appears to be transcribed at a low level under $\mathrm{N}^{+}$conditions, but it is not essential for vegetative growth because a null mutation in het $R$ is not lethal. Upon transfer to $\mathrm{N}^{-}$conditions, the abundance of the het $R$ transcript increases rapidly within $6 \mathrm{hr}$, consistent with an early role for the het $R$ protein.

het $R$ gene function in Anabaena 7120 is similar in some respects to that of the whiG gene of Streptomyces coelicolor (Chater et al. 1989) and the brlA gene of Aspergillus nidulans (Adams et al. 1988). Interruption of the whiG gene prevents sporulation but has no effect on vegetative growth. Likewise, brlA mutants are defective in the later stages of conidiophore development, but not in vegetative growth. The sequence of the whiG product is similar to that of several Bacillus subtilis sigma factors but not to that of four other $S$. coelicolor sigma factors. Greatest similarity is not with $B$. subtilis sigma factors required for sporulation, but with $\sigma^{D}\left(\sigma^{28}\right)$, used for transcription of genes involved in motility and chemotaxis (Helmann et al. 1988). In contrast, BrlA contains two zinc finger motifs, indicative of transcriptional regulatory function (Adams et al. 1988). Extra copies of the wild-type whiG gene result in a hypersporulation phenotype that can be reversed by introduction of multiple copies of a promoter recognized by $\sigma^{\mathrm{D}}$. Similarly, misscheduled expression of brlA results in cessation of vegetative growth and the function of conidiospores.

The presence of extra copies of het $R$ in Anabaena 7120 has deleterious consequences. Under $\mathrm{N}^{-}$conditions, it leads to supernumerary heterocysts, which may develop as arrays of two or more adjacent heterocysts. Under other circumstances it also appears to be detrimental, resulting in the accumulation of mutations in the cloned het $R$ gene or possibly in other genes that participate in the commitment of heterocysts to differentiation. In the isolation of wild-type genes by mutant complementation, the isolated het $R$ gene was occasionally mutated by the time it was recovered in E. coli. Presumably these mutations arose by genetic exchange with the mutated chromosomal copy of het $R$. Because the same excessive heterocyst phenotype is observed in wild-type Anabaena 7120 after introduction of het $R$ on a plasmid, it may be possible to identify new genes whose products are required for differentiation by selecting $\mathrm{Fix}^{-}$survivors from such plasmid-containing strains.

The cellular interactions governing the pattern of heterocyst differentiation have been reviewed recently (Wolk 1989). Based on the early observations of Wolk that mechanical disruption of filaments led to an increase of heterocyst frequency (Wolk 1967), subsequent work of Wilcox on mechanical destruction of individual proheterocysts (Wilcox et al. 1973b), and that of Meeks on the metabolic path of nitrogen following fixation of $\mathrm{N}_{2}$ (Meeks et al. 1978), the following picture of pattern formation emerged: heterocysts are the source of an inhibitor of differentiation, possibly glutamine or a derivative, that diffuses along the filament from cell to cell and is metabolized by vegetative cells. When the level of inhibitor falls below a threshold in a candidate cell, that cell initiates differentiation. A certain minimum number of adjacent vegetative cells must remain to provide support for the developing heterocyst, otherwise it will cease development and revert to a vegetative cell. In strains such as Anabaena 7120 that do not typically form multiple heterocysts under free-living conditions, heterocysts that begin to form as pairs compete with each other until one becomes a heterocyst and the other regresses to a vegetative cell.

Our results shed no light on the questions of candidate cells or the nature of the inhibitor. With regard to the mechanism by which het $R$ regulates differentiation, the het $R$ gene product could act positively in developing cells or negatively in vegetative cells. Since mutant 216 and the null mutant WJB313 do not differentiate under any conditions, we believe that the het $R$ product acts positively in developing cells. These mutants bleach rapidly under nitrogen starvation conditions, so het $R$ is not required for the activation of the protease(s) that digest the phycobiliproteins (Wood and Haselkorn 1980). The het $R$ gene could encode a different protease (with serine179 as part of its active site) but the sequence data base searches have thus far failed to find similarity with known proteases.

The supernumerary heterocyst phenotype of the multicopy het $R$ strains is consistent with positive action of the gene product. One could envision the wild-type het $R$ gene product as a necessary activator of differentiation unless it binds a nitrogen-containing inhibitor, such as glutamine. Inactivation or titration of the het $R$ gene product with inhibitor would result in the absence of activation ability. In the extra-copy het $R$ strain there would be insufficient inhibitor to titrate all the het $R$ product in many of the cells, and these would differentiate. Effectively, the extra copies of het $R$ would raise the threshold of inhibitor concentration below which differentiation is initiated. Thus, the differentiation unit would sometimes become not a single cell, but a group of cells, and the spacing between heterocysts would narrow. In support of this idea is the evidence that heterocyst numbers in strains containing extra copies of het $R$ are correlated with the level of inhibition of heterocyst development afforded by different nitrogen sources. The lowest frequency of heterocysts $(\sim 5 \%)$ is seen when ammonia is used as the nitrogen source, which is correlated with the ability of ammonia to repress heterocyst development completely in wild-type Anabaena 7120. During growth with nitrate, extra-copy het $R$ strains produce 5$10 \%$ heterocysts, consistent with the observation that wild-type Anabaena is not completely repressed by nitrate, typically forming up to $1 \%$ heterocysts in our cultures. Finally, under completely derepressing conditions 
when no fixed nitrogen is present, extra-copy het $R$ strains produce supernumerary heterocysts at $10-25 \%$, compared to the typical $5-10 \%$ frequency of heterocysts in wild-type Anabaena.

There are alternative hypotheses for the role of the het $R$ gene product in heterocyst differentiation. Even though het $R$ does not show significant sequence similarity to known sigma factors, its effect on heterocyst differentiation could be explained if the Anabaena cells are both dependent on its function for differentiation and sensitive to the levels of its product, as might occur if het $R$ interacted with RNA polymerase as a transcriptional activator for differentiation. The inappropriate expression of such an activator might lead to the differentiation of cells at an improper place in the cell cycle, affording an explanation for the occasional appearance of tightly apposed and incompletely divided heterocysts.

Another possibility is that the het $R$ gene product might be more indirectly involved in differentiation, perhaps by measuring the ratio of fixed carbon to nitrogen in the cell. Here one could envision the lack of het $R$ to result in the "sensing" of a low carbon to nitrogen ratio. Because it has been shown that heterocysts require the support of a minimum number of adjacent vegetative cells (Wilcox et al. 1973b), it is likely that filaments with low fixed-carbon reserves will not effectively differentiate heterocysts. Thus, het $R$ mutant cells might act as if chronically starved for fixed carbon and not differentiate heterocysts under any conditions. Conversely, in the presence of excess gene product, an abnormally high ratio of carbon to nitrogen might be sensed, resulting in the production of excessive heterocysts. Such a general function for het $R$ might explain the presence of het $R$ hybridizing DNA in nonheterocystous nitrogen-fixing strains of cyanobacteria.

Multiple heterocysts have been seen before in cyanobacteria. Supernumerary heterocysts appear when Anabaena is grown in the presence of subinhibitory concentrations of rifamycin (Wolk and Quine 1975) or in the presence of the tryptophan analog 7-azatryptophan (Mitchison and Wilcox 1973). In neither of these cases has a mechanism been proposed for the observed behavior. Mutants of Anabaena cylindrica have been described that produce supernumerary grouped heterocysts constitutively (Wilcox et al. 1975). In several symbioses, the frequency of heterocysts is considerably elevated over the frequency seen in the free-living state (Hill 1975; Peters et al. 1980). In these cases and in our experience with extra-copy het $R$, the pattern of heterocyst formation is altered, and multiple adjacent heterocysts are often formed along the filament. Heterocysts are often more closely spaced in general, leading to an overall increase in heterocyst numbers. It is plausible that relatively simple changes in signal generation or reception can account for these observations.

\section{Materials and methods}

Strains and culture conditions

Many of the strains of cyanobacteria mentioned here have been previously described (Rippka et al. 1979; Schneider and Haselkorn 1988; Buikema and Haselkorn 1991). Other strains were received from the following sources: Anabaena $7118 \mathrm{Rev}$, a $\mathrm{Fix}^{+}$revertant of PCC 7118, and Synechocystis PCC 6803 from R. Rippka at the Institut Pasteur; Nostoc 7911 Mac R1 and R2, two independent Fix ${ }^{+}$revertants of Nostoc 7911 Mac, and Nostoc 7801, from J. Meeks at the University of California at Davis; Anabaena M-131 and Nostoc ATCC 29107 from C.P. Wolk at Michigan State University; Anabaena 77 S15 from C. Franche at the Institut Pasteur; Anabaena CA ATCC 33047 from F.R. Tabita at Ohio State University; and Synechocystis PCC 6714 from F. Joset at the Faculté des Sciences Luminy, Marseille.

For liquid culture, Anabaena strains were grown in a modified Kratz and Myers medium C (K\&M) or BG-11 medium (Kratz and Myers 1955; Rippka et al. 1979). The modified K\&M medium contained $1.125 \mathrm{mM} \mathrm{Na}_{2} \mathrm{HPO}_{4}$ and $\mathrm{K}_{2} \mathrm{HPO}_{4}$ each in place of the $\mathrm{Na}_{2} \mathrm{HPO}_{4}$, and typically contained 3-5 mM MOPS buffer ( $\mathrm{pH} 7.5$ ). For $\mathrm{N}^{+}$growth, K\&M was supplemented with $2.5 \mathrm{mM}(\mathrm{NH} 4)_{2} \mathrm{SO}_{4}$, and BG-11 with $17.6 \mathrm{mM} \mathrm{NaNO}$. For growth on plates, $1.3 \%$ agar (BBL purified) was added prior to autoclaving. Cultures were typically grown under $30-40$ $\mu \mathrm{E} / \mathrm{m}^{2} / \mathrm{sec}$ cool white fluorescent lighting at $25-30^{\circ} \mathrm{C}$ in the presence of $2 \% \mathrm{CO}_{2}$ (large-scale liquid cultures were bubbled with a $2 \% \mathrm{CO}_{2}$-air mixture). For selective growth of Anabaena 7120 strains, media were supplemented with $25-30 \mu \mathrm{g} / \mathrm{ml} \mathrm{neo-}$ mycin. Mid-log phase cells refer to cultures containing 2-6 $\mu \mathrm{g} / \mathrm{ml}$ of chlorophyll corresponding to $0.7 \times 10^{7}$ to $2.0 \times 10^{7}$ cells $/ \mathrm{ml}$.

E. coli strains were typically grown in Luria broth (LB) (Maniatis et al. 1982 ) for liquid culture, and LB solidified with $1.3 \%$ agar for plate culture. For selective growth, media were supplemented with antibiotics at the following concentrations: 100 $\mu \mathrm{g} / \mathrm{ml}$ ampicillin or carbenicillin, $50 \mu \mathrm{g} / \mathrm{ml}$ kanamycin, and 10 $\mu \mathrm{g} / \mathrm{ml}$ chloramphenicol. For plasmid isolation, E. coli strains were grown in LB supplemented with M9 salts (Maniatis et al. 1982), 2 g/liter glucose, 1 g/liter sodium succinate, and the appropriate antibiotic.

\section{Molecular biological techniques}

General molecular biological techniques were as described (Maniatis et al. 1982). Plasmid isolation from E. coli was by the alkaline lysis method.

Sequencing templates were prepared from the insert of pWB216S2.4 by digestion with Pst I, resulting in a $3.2-\mathrm{kb}$ fragment containing the $2.4-\mathrm{kb}$ Sau3AI insert joined to the $3^{\prime}$ end of the neo gene from pDUCA7 (Buikema and Haselkorn 1991). The $3.2-\mathrm{kb}$ fragment was ligated into the Pst site of pUC19, and two plasmids containing the insert in opposite orientations were obtained. One of these, pWB19-7, was used for sequencing without further modifications. The other plasmid was digested with SmaI to release most of the fragment of pDUCA7, followed by religation to form $\mathrm{pWB} 19-12$. Several sequencing templates / see below) with deletions of varying lengths $5^{\prime}$ or $3^{\prime}$ to the het $R$ ORF were selected for subcloning into an Anabaena shuttle vector. The inserts from these deletion plasmids were removed using $P_{v u I I}$ and ligated into SmaI-digested pCCB110. pCCB 110 is a shuttle vector based on pRL25C (Elhai and Wolk 1988) which has had the polylinker from pIC20R (Marsh et al. 1984) ligated into the EcoRI site (C. Bauer, pers. comm.).

\section{Northern and Southern hybridizations}

DNA of many of the cyanobacterial strains was provided by Martin E. Mulligan (Memorial University of Newfoundland). DNA was prepared from the remaining strains as previously 
described (Golden et al. 1985). A Northern blot of total RNA (10 $\mu \mathrm{g}$ per lane) from Anabaena 7120 isolated at 6 -hr intervals from an $\mathrm{N}^{-}$induced culture as described (Golden et al. 1987) was obtained from Bianca Brahamsha. Northern blot analysis was performed as described (Maniatis et al. 1982). Southern blot analysis was performed as described (Reed and Mann 1985) using 1-2 $\mu \mathrm{g}$ of DNA per lane.

\section{DNA sequence analysis}

Sequencing deletions were constructed using an Erase-a-Base kit from Promega Biotech on SstI- and BamHI-digested pWB197 and SstI- and SmaI-digested pWB19-12. Sequences were determined by the chain-termination method (Sanger et al. 1977) using Sequenase (United States Biochemical Corporation) on double-stranded template DNA using a modification of a published method (Kraft et al. 1988). Essentially the ethanol precipitation step after RNase digestion was modified to use 0.6 volumes of isopropanol at room temperature to avoid precipitating RNA oligonucleotides, and the subsequent PEG precipitation was omitted. Buffer gradient sequencing gels were prepared and run as described (Sheen and Seed 1988). Both strands were completely determined, and the sequence is available from GenBank under accession number M37779. DNA sequence was analyzed using the University of Wisconsin Genetic Computer Group's Sequence Software Package and programs written by one of us (W.J.B.) for the Apple Macintosh.

\section{PCR techniques}

Techniques for the amplification of selected DNA sequences by polymerase chain reaction (PCR) were essentially as described in the manual accompanying the GeneAmp kit (Perkin Elmer Cetus). A 318-bp region from strain 216 and wild-type Anabaena 7120 DNA was amplified using the following two 26 base flanking oligonucleotides: 5'-CCAGGGCGAT CGCGCATTCC CGGTAC-3' and 5'-GTCCGCTCTT GGTCGTCTGC TGGGGC-3'. Following symmetric amplification, approximately $0.1 \mu \mathrm{g}$ of the double-stranded product was asymmetrically amplified using a $50: 1$ ratio of one oligonucleotide to the other. Both strands were amplified by reversing the ratio of oligonucleotides. The resultant single-stranded DNA was separated from excess oligonucleotides by passage through a Sephacryl 300 column and sequenced as described above using the appropriate 26-base oligonucleotide primer.

\section{Construction of the hetR insertion mutant WIB313}

Anabaena strain WJB313, in which the genomic het $R$ gene is interrupted by a neomycin-resistance cassette, was constructed as follows. The neo gene of Tn5 driven by the Anabaena 7120 woxA promoter (Borthakur and Haselkorn 1989) from pDB13 (Borthakur et al. 1990) was removed using SmaI, HindIII, and ScaI. The wox $A$ gene encodes the water-splitting protein of photosystem II; its promoter is constitutive in Anabaena 7120. Following end repair with Klenow fragment, this DNA was ligated into the end-repaired $X b a I$ site of the het $R$ gene in pWB1912 to form pWB19-12K. The entire interrupted het $R$ gene was isolated by digestion of pWB19-12K with SstI and SalI and ligated into SstI- and XhoI-digested pRL271 [which contains the B. subtilis $\operatorname{sacB}$ gene (Cai and Wolk 1990), and was kindly provided by Y. Cai and C.P. Wolk\| to form pWB19-12KS. After transformation of E. coli MC1061, resultant colonies were verified for sensitivity to $5 \%$ sucrose on LB plates due to the presence of the $s a c B$ gene. pWB19-12KS was then used to transform MC1061/pRL528 for conjugation into wild-type Anabaena
7120 , as described above. Single recombinant colonies in which the entire pWB19-12KS had integrated into the genomic copy of het $R$ were identified after selection on $\mathrm{BG}-11 \mathrm{~N}^{+}$plates containing $30 \mu \mathrm{g} / \mathrm{ml}$ neomycin for 2-3 weeks. Double recombinants in which a single interrupted copy of het $R$ remained in the genome were isolated by placing $\sim 5 \times 10^{7}$ single recombinant cells on BG-11 $\mathrm{N}^{+}$plates containing $5 \%$ sucrose and 30 $\mu \mathrm{g} / \mathrm{ml}$ neomycin. Several single colonies were selected and seen to have the same phenotype as strain 216 . Southern hybridization to genomic DNA digests from the presumptive single and double recombinants demonstrated the expected restriction patterns, indicative of two copies of the het $R$ gene fone interrupted) and plasmid sequences in the single recombinants, and one copy of the interrupted het $R$ gene in the double recombinants (data not shown).

\section{Microscopy}

Photomicroscopy of Anabaena cells was performed on a Zeiss Axiophot microscope equipped with phase-contrast and Nomarsky (DIC) optics. Photomicrographs were taken using Kodak Technical Pan film at 50 ASA.

\section{Acknowledgments}

This work was supported by grants from the National Institutes of Health (GM21823 and GM40685) and a fellowship to W.J.B. from the American Cancer Society. We thank Bianca Brahamsha for a Northern blot, Martin Mulligan for cyanobacterial DNA samples, Conrad Halling, Bianca Brahamsha, Kristin Bergsland, and Helen Revel for critical reading of the manuscript, and Jim Shapiro for assistance with the light microscopy.

The publication costs of this article were defrayed in part by payment of page charges. This article must therefore be hereby marked "advertisement" in accordance with 18 USC section 1734 solely to indicate this fact.

\section{References}

Adams, T.H., M.T. Boylan, and W.E. Timberlake. 1988. brlA is necessary and sufficient to direct conidiophore development in Aspergillus nidulans. Cell 54: 353-362.

Bairoch, A. 1989. PROSITE: A dictionary of protein sites and patterns, 5 th ed. University of Geneva, Geneva.

Böhme, H. and R. Haselkorn. 1988. Molecular cloning and nucleotide sequence analysis of the gene coding for heterocyst ferredoxin from the cyanobacterium Anabaena sp. strain PCC 7120. Mol. Gen. Genet. 214: 278-285.

Borthakur, D. and R. Haselkorn. 1989. Nucleotide sequence of the gene encoding the $33 \mathrm{kDa}$ water oxidizing polypeptide in Anabaena sp. strain PCC 7120 and its expression in Escherichia coli. Plant Mol. Biol. 13: 427-439.

Borthakur, D., M. Basche, W.J. Buikema, P.B. Borthakur, and R. Haselkorn. 1990. Expression, nucleotide sequence and mutational analysis of two open reading frames in the nif gene region of Anabaena sp. strain PCC 7120. Mol. Gen. Genet. 221: $227-234$.

Buikema, W.J. and R. Haselkorn. 1991. Isolation and complementation of nitrogen fixation mutants of the cyanobacterium Anabaena sp. strain PCC 7120. I. Bacteriol. (in press).

Cai, Y. and C.P. Wolk. 1990. Use of a conditionally lethal gene in Anabaena sp. strain PCC 7120 to select for double recombinants and to entrap insertion sequences. J. Bacteriol. 172: 3138-3145.

Carr, N.G. 1983. Biochemical aspects of heterocyst differentia- 
tion and function. In Photosynthetic prokaryotes (ed. G.C. Papageorgiou and L. Packer), pp. 265-280. Elsevier Science Publishing, New York.

Chater, K.F., C.J. Bruton, K.A. Plaskitt, M.J. Buttner, C. Méndez, and J.D. Helmann. 1989. The developmental fate of $S$. coelicolor hyphae depends upon a gene product homologous with the motility $\sigma$ factor of $B$. subtilis. Cell 59: 133-143.

Chou, P.Y. and G.D. Fasman. 1978. Prediction of the secondary structure of proteins from their amino acid structure. Adv. Enzymol. 47: 45-148.

Elhai, J. and C.P. Wolk. 1988. Conjugal transfer of DNA to cyanobacteria. Methods Enzymol. 167: 747-754.

Garnier, J., D.J. Osguthorpe, and B. Robson. 1978. Analysis of the accuracy and implications of simple methods for predicting the secondary structure of globular proteins. J. Mol. Biol. 120: $97-120$.

Golden, J.W., S.J. Robinson, and R. Haselkorn. 1985. Rearrangement of nitrogen fixation genes during heterocyst differentiation in the cyanobacterium Anabaena. Nature 314: 419423.

Golden, J.W., M.E. Mulligan, and R. Haselkorn. 1987. Different recombination site specificity of two developmentally regulated genome rearrangements. Nature 327: 526-529.

Haselkorn, R. 1978. Heterocysts. Annu. Rev. Plant Physiol. 29: 319-344.

Helmann, J.D., L.M. Marquez, and M.J. Chamberlin. 1988. Cloning, sequencing and disruption of the Bacillus subtilis $\sigma^{28}$ gene. I. Bacteriol. 170: 1568-1574.

Hill, D. 1975. The pattern of development of Anabaena in the Azolla-Anabaena symbiosis. Planta 122: 179-184.

Kraft, R., J.J. Tardiff, K.S. Krauter, and L.A. Leinwand. 1988. Using mini-prep plasmid DNA for sequencing double stranded templates with sequenase. BioTechniques 6: 544 547

Kratz, W.A. and J. Myers. 1955. Nutrition and growth of several blue-green algae. Am. I. Bot. 42: 282-287.

Kustu, S., E. Santero, J. Keener, D. Popham, and D. Weiss. 1989. Expression of $\sigma^{54}$ (ntrA)-dependent genes is probably united by a common mechanism. Microbiol. Rev. 53: 367-376.

Maniatis, T., E.F. Fritsch, and J. Sambrook. 1982. Molecular cloning: A laboratory manual. Cold Spring Harbor Laboratory, Cold Spring Harbor, New York.

Marsh, J.L., M. Erfle, and E.J. Wykes. 1984. The pIC plasmid and phage vectors with versatile cloning sites for recombinant selection by insertional inactivation. Gene 32: 481-485.

Meeks, J.C., C.P. Wolk, W. Lockau, N. Schilling, P.W. Shaffer, and W.S. Chien. 1978. Pathways of assimilation of $\left[{ }^{13} \mathrm{~N} \mid \mathrm{N}_{2}\right.$ and ${ }^{13} \mathrm{NH}_{4}$ l by cyanobacteria with and without heterocysts. I. Bacteriol. 134: 125-130.

Mitchison, G.J. and M. Wilcox. 1973. Alteration in heterocyst pattern of Anabaena produced by 7-azatryptophan. Nat. New Biol. 246: 229-233.

Nierzwicki-Bauer, S.A., S.E. Curtis, and R. Haselkorn. 1984. Cotranscription of genes encoding the small and large subunits of ribulose-1,5-bisphosphate carboxylase in the cyanobacterium Anabaena 7120. Proc. Nat1. Acad. Sci. 81: 59615965.

Pearson, W.R. and D.J. Lipman. 1988. Improved tools for biological sequence comparison. Proc. Natl. Acad. Sci. 85: 2444-2448.

Peters, G.A., T.B. Ray, B.C. Mayne, and R.E. Toia Jr. 1980. Azolla-Anabaena association: Morphological and physiological studies. In Nitrogen fixation (ed. W.E. Newton and W.H. Orme-Johnson), vol. II, pp. 293-309. University Park Press, Baltimore.

Reed, K.C. and D.A. Mann. 1985. Rapid transfer of DNA from agarose gels to nylon membranes. Nucleic Acids Res. 13: 7207-7221.

Rice, D., B.J. Mazur, and R. Haselkorn. 1982. Isolation and physical mapping of nitrogen fixation genes from the cyanobacterium Anabaena 7120. J. Biol. Chem. 257: 13157-13163.

Rippka, R., J. Deruelles, J.B. Waterbury, M. Herdman, and R.Y. Stanier. 1979. Genetic assignments, strain histories and properties of pure cultures of cyanobacteria. J. Gen. Microbiol. 111: 1-61.

Sanger, F., S. Nicklen, and A.R. Coulson. 1977. DNA sequencing with chain-terminating inhibitors. Proc. Natl. Acad. Sci. 74: 5463-5467.

Schneider, G.J. and R. Haselkorn. 1988. RNA polymerase subunit homology among cyanobacteria, other eubacteria and archaebacteria. J. Bacteriol. 170: 4136-4140.

Sheen, J. and B. Seed. 1988. Electrolyte gradient gels for DNA sequencing. BioTechniques 6: 942-944.

Stewart, W.D.P. 1980. Some aspects of structure and function in $\mathrm{N}_{2}$-fixing cyanobacteria. Annu. Rev. Microbiol. 34: 497-536.

Wilcox, M., G.J. Mitchison, and R.J. Smith. 1973a. Pattern formation in the blue-green alga, Anabaena. I. Basic mechanisms. I. Cell Sci. 12: 707-723.

- 1973b. Pattern formation in the blue-green alga, Anabaena. II. Controlled proheterocyst regression. I. Cell Sci. 13: $637-649$.

. 1975. Mutants of Anabaena cylindrica altered in heterocyst spacing. Arch. Microbiol. 103: 219-223.

Winkenbach, F. and C.P. Wolk. 1973. Activities of enzymes of the oxidative and reductive pentose phosphate pathways in heterocysts of a blue-green alga. Plant Physiol. 52: 480-483.

Wolk, C.P. 1967. Physiological basis of the pattern of vegetative growth of a blue-green alga. Proc. Natl. Acad. Sci. 57: 12461251 .

1968. Movement of carbon from vegetative cells to heterocysts in Anabaena cylindrica. I. Bacteriol. 96: 21382143.

1982. Heterocysts. In The biology of cyanobacteria led. N.G. Carr and B.A. Whitton|, pp. 359-386. Blackwell Scientific Publishers, London.

-1989. Alternative models for the development of the pattern of spaced heterocysts in Anabaena (Cyanophyta). Plant Syst. Evol. 164: 27-31.

Wolk, C.P. and M.P. Quine. 1975. Formation of onedimensional patterns by stochastic processes and by filamentous blue-green algae. Dev. Biol. 46: 370-382.

Wood, N.B. and R. Haselkorn. 1980. Control of phycobiliprotein proteolysis and heterocyst differentiation in Anabaena. 1 . Bacteriol. 141: 1375-1385. 


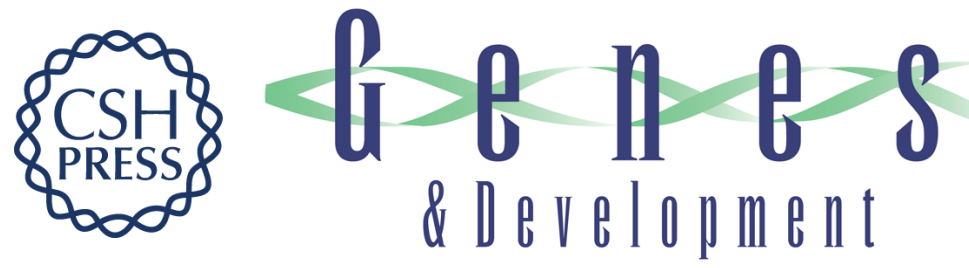

\section{Characterization of a gene controlling heterocyst differentiation in the cyanobacterium Anabaena 7120.}

W J Buikema and R Haselkorn

Genes Dev. 1991, 5:

Access the most recent version at doi:10.1101/gad.5.2.321

References This article cites 38 articles, 15 of which can be accessed free at:

http://genesdev.cshlp.org/content/5/2/321.full.html\#ref-list-1

License

Email Alerting

Service

Receive free email alerts when new articles cite this article - sign up in the box at the top right corner of the article or click here.

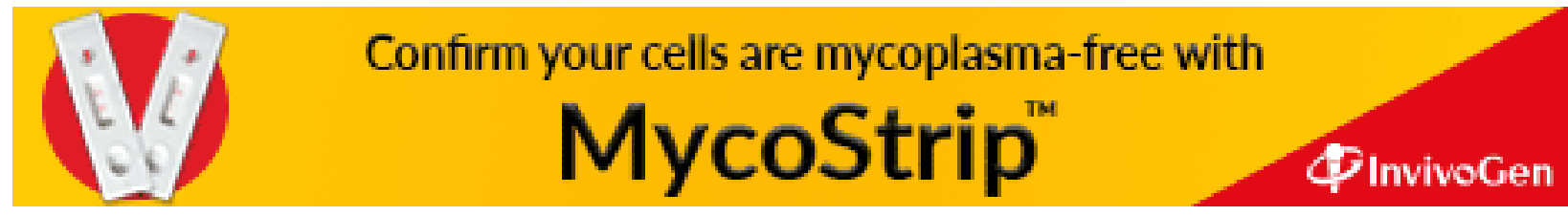

